\title{
Interception du rayonnement utile à la photosynthèse chez la luzerne : variations et modélisation
}

Ghislain GOSSE, Michel CHARTIER, Claude VARLET-GRANCHER $\left({ }^{*}\right)$ \& Raymond BONHOMME (*)

I.N.R.A., Station de Bioclimatologie, Route de St-Cyr, F 78000 Versailles.

(*) I.N.R.A., Laboratoire de Bioclimatologie, Estrées-Mons, F 80200 Peronne.

\section{RÉSUMÉ}

\section{Luzerne,}

Rayonnement intercepté, Modèle.

\begin{abstract}
Une étude détaillée de l'efficience d'interception du rayonnement photosynthétiquement actif d'une culture de luzerne montre que, pour une même surface foliaire, on ne peut observer de différence entre les quatre pousses ou repousses de la luzerne.

Parallèlement à cette approche expérimentale, un modèle de pénétration du rayonnement dans la culture conduit à des résultats similaires.

L'effet de la structure de la culture de luzerne sur l'efficience d'interception ne peut donc expliquer les différences de production observées entre le printemps et l'automne.
\end{abstract}

\section{SUMMARY}

Alfalfa, Intercepted radiation, Model.

\section{Photosynthetically active radiation by alfalfa: Variations and modeling}

A study of photosynthetically active radiation trapping efficiency has been made on different growths and regrowths of alfalfa over three years. This study shows that for the same leaf area index, the PAR trapping efficiency is not affected by the period of regrowth of alfalfa, so that a simple relation can be used for PAR trapping efficiency versus LAI :

$$
\varepsilon \varphi_{\mathrm{i}}=0.97\left(1-\mathrm{e}^{-0.88 \mathrm{LAl}}\right) \text {. }
$$

In relation to this cxperimental approach, a model of penetration of PAR through the canopy has been adapted for this crop. Similar results have been obtained.

Experimental measurements and data from our model for the ratio between PAR and total transmittance of the crop have been compared with data from other authors (ANDERSON, 1969 and KUMURA, 1969) on other crops. An effect of leaf inclination is given to explain the differences observed between the different crops. The effect of vegetation structure on PAR trapping efficiency cannot explain differences of production observed between spring and autumn.

\section{INTRODUCTION}

La luzerne (Medicago sativa L.) se caractérise, en climat océanique et en absence de contrainte hydrique, par une production de matière sèche exportable (feuilles + tiges) variant en moyenne de $6 \mathrm{~T} /$ ha pour la pousse de printemps à $2 \mathrm{~T} /$ ha pour la repousse d'automne. Différentes hypothèses peuvent être envisagées pour expliquer cette réduction de production en cours d'année :

- Réduction de la masse de carbone fixé par la culture au cours de la période de végćtation; cette réduction peut avoir d'une part une origine climatique (rayonnement incident et températures moins favorables à l'automne qu'au printemps) et d'autre part une origine biologique (la structure de la végétation, la surface foliaire ou la réponse intrinsèque de la feuille peuvent être différentes suivant le moment de l'année).

- Modification de la répartition des assimilats au cours de la période séparant deux coupes (DEMARLY, 1957) et d'une repousse à l'autre entre les parties aériennes et les racines (HuNT et al., 1970 ; Gosse et al., 1981).

Pour essayer d'expliquer les différences de production observées entre l'automne et le printemps, nous nous sommes intéressés au facteur rayonnement et plus précisément au rayonnement intercepté par la culture en tant que paramètre explicatif du bilan de carbone de cette culture pendant la phase diurne. Après une description du dispositif et des méthodes, nous présenterons les variations du rayonnement intercepté, tant au cours d'une repousse que d'une repousse à l'autre, ainsi que la modélisation que nous avons adaptée à ce couvert végétal. 


\section{TABLEAU 1}

Liste des symboles.

List of symbols.

\begin{tabular}{|c|c|}
\hline $\mathrm{D} / \mathrm{G}$ & : Rapport Diffus/Global du rayonnement incident. \\
\hline$\varepsilon_{\mathrm{i}}$ & $\begin{array}{l}\text { Efficience dinterception pour le rayonnement solaire } \\
\text { global. }\end{array}$ \\
\hline$\varepsilon \varphi_{i}$ & $\begin{array}{l}\text { Efficience d'interception pour le rayonnement photo- } \\
\text { synthétiquement actif PAR. }\end{array}$ \\
\hline $\mathrm{G}$ & : Rayonnement solaire global $[400-2400 \mathrm{~nm}]\left(\mathrm{W} . \mathrm{m}^{-2}\right)$. \\
\hline PAR & $\begin{array}{l}\text { : Rayonnement solaire photosynthétiquement actif } \\
{[400-700 \mathrm{~nm}]\left(\mu \text { mole } \cdot \mathrm{m}^{-2} \cdot \mathrm{s}^{-1}\right) \text {. }}\end{array}$ \\
\hline $\mathbf{R}$ & $\begin{array}{l}\text { Rayonnement }\left(\mathrm{W} \cdot \mathrm{m}^{-2}\right) . \\
\text { a, index pour absorbé ex }: \mathrm{G}_{\mathrm{a}}, \mathrm{PAR}_{\mathrm{a}}, \mathrm{R}_{\mathrm{a}} \text {. } \\
\mathrm{i} \text {, index pour incident ex: } \mathrm{G}_{\mathrm{i}}, \mathrm{PAR}_{\mathrm{i}}, \mathrm{R}_{\mathrm{j}} \text {. } \\
\mathrm{r} \text {, index pour réfléchi ex: } \mathrm{G}_{\mathrm{r}}, \mathrm{PAR}_{\mathrm{r}}, \mathrm{R}_{\mathrm{r}} \text {. } \\
t \text {, index pour transmis ex: } \mathrm{G}_{\mathrm{t}}, \mathrm{PAR}_{\mathrm{t}}, \mathrm{R}_{\mathrm{t}} \text {. }\end{array}$ \\
\hline $\mathrm{r}$ & : Coefficient de réflexion de la culture (albédo). \\
\hline$r_{\varphi}$ & : Coefficient de réflexion de la culture dans le PAR. \\
\hline$r_{s}$ & : Coefficient de réflexion du sol. \\
\hline $\mathrm{r} \varphi_{\mathrm{s}}$ & : Coefficient de réflexion du sol dans le PAR. \\
\hline$\rho$ & : Coefficient de réflexion de la feuille. \\
\hline $\mathrm{S}_{\mathrm{s}}$ & : Durée d'insolation journalière. \\
\hline$\tau$ & : Cocfficient de transmission de la feuille. \\
\hline $\mathrm{t}$ & $\begin{array}{l}\text { : Coefficient de transmission de la culture pour le } \\
\text { rayonnement solaire global. }\end{array}$ \\
\hline$t \varphi$ & $\begin{array}{l}\text { Coefficient de transmission de la culture pour le } \\
\text { rayonnement solaire photosynthétiquement actif. }\end{array}$ \\
\hline
\end{tabular}

\section{MATÉRIEL ET MÉTHODES}

L'expérience a été réalisée sur le site de La Minière $\left(49^{\circ} \mathrm{N}, 2^{\circ} \mathrm{W}\right)$ sur une culture de luzerne (variété « du Puits») pendant 3 années (1978-80).

Le bilan radiatif d'une culture homogène peut s'écrire (AlleN \& RichaRdSON, 1968 ; VARLET-GRANCHER et al., 1982), (cf. tabl. 1):

$$
R_{a}=R_{i}-R_{r}-\left(1-r_{s}\right) \cdot R_{t}
$$

soit :

- Pour le rayonnement solaire [400-2 $400 \mathrm{~nm}$ ] exprimé en énergie $\left(W \cdot \mathrm{m}^{-2}\right)$

$$
\mathrm{G}_{\mathrm{a}}=\mathrm{G}_{\mathrm{i}}-\mathrm{G}_{\mathrm{r}}-\left(1-\mathrm{r}_{\mathrm{s}}\right) \mathrm{G}_{\mathrm{t}} .
$$

- Pour le rayonnement utile à la photosynthèse [400$700 \mathrm{~nm}$ ] exprimé en flux de photons $\left(\mu\right.$ mole $\left.\cdot \mathrm{m}^{-2} \cdot \mathrm{s}^{-1}\right)$

$$
\mathrm{PAR}_{\mathrm{a}}=\mathrm{PAR}_{\mathrm{i}}-\mathrm{PAR}_{\mathrm{r}}-\left(1-\mathrm{r} \varphi_{\mathrm{s}}\right) \mathrm{PAR}_{\mathrm{l}} .
$$

Les rayonnements absorbés $G_{a}, P_{a}$ peuvent être rapportés au rayonnement incident, nous obtenons respectivement :

$$
\begin{aligned}
\varepsilon_{i} & =\frac{G_{a}}{G_{i}}=1-r-\left(1-r_{s}\right) t \\
\varepsilon \varphi_{i} & =\frac{\operatorname{PAR}_{a}}{\operatorname{PAR}_{i}}=1-r \varphi-(1-r \varphi v) t \varphi .
\end{aligned}
$$

Les mesurcs de rayonnement étaient réalisées au moyen des instruments suivants :

- capteurs Li-Cor (Quantum Sensor) pour la mesure du rayonnement incident utile à la photosynthèse $\mathrm{PAR}_{\mathrm{i}}$ et réfléchi utile à la photosynthèse $\mathrm{PAR}_{\mathrm{r}}$;

- pyranomètre Kipp et Zonen pour la mesure du rayonnement global incident $\mathrm{G}_{i}$;
- pyranomètres linćaires (type INRA) disposés en série pour la mesure du rayonnement global transmis $G_{t}$.

Les paramètres précédents étaient intégrés sur une heure et pour la présente étude nous avons utilisé ces données sommées sur la journée.

- Le rayonnement transmis utile à la photosynthèse $\mathrm{PAR}_{1}$ a été estimé à partir d'une relation entre le rapport t $\varphi / t$ et l'indice foliaire LAI. Cette relation a été établie sur la même variété de luzerne; le $\operatorname{PAR}_{t}$ était alors mesuré à l'aide d'un capteur Li-Cor disposé à $7 \mathrm{~cm}$ aul-dessus du sol sur un système d'entraînement à vitesse constante d'une longucur de $1,20 \mathrm{~m}$.

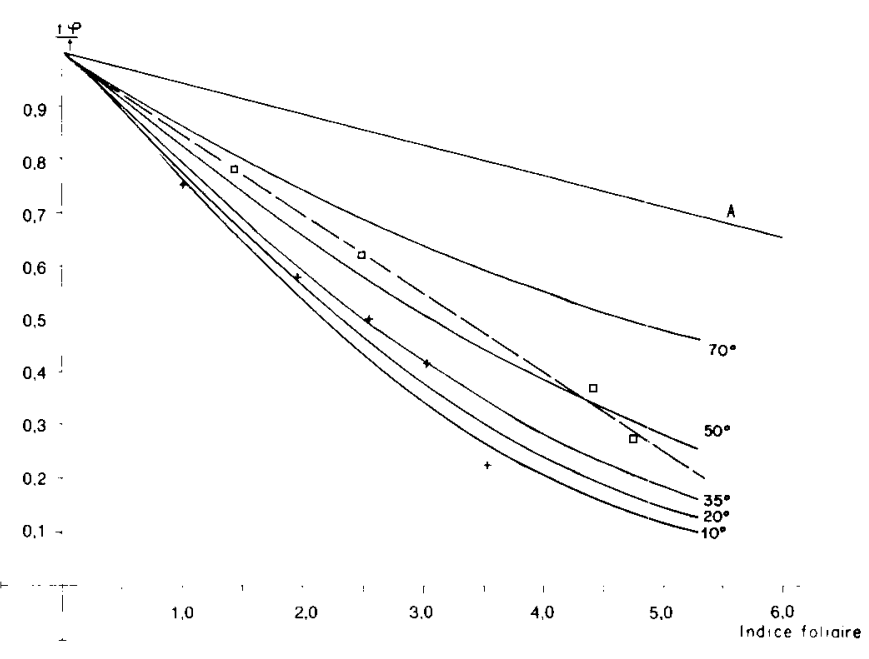

Figure 1

Evolution du rapport $\mathrm{t} \varphi / \mathrm{t}$ en fonction de l'indice foliaire If: Points expérimentaux pour luzerne

Points expérimentaux pour soja, d'après KuMURA (1969).

(Courbe A). Ajustement d'après ANDERSON (1969) pour du blé.

Résultats du modèle pour différentes inclinaisons de feuilles $10,20,35,50,70^{\circ}$.

$\mathrm{t} \varphi / \mathrm{t}$ ratio in relation to leaf area index :

alfalfa experimental data.

soybean experimental data from KUMURA (1969).

(curve A) fitted from wheat data ANDERSON (1969).

results from model for different leaf inclinations : 10, 20, 35, $50,70^{\circ}$.

La figure 1 représente cette relation. Les points expérimentaux obtenus sur luzerne ont été ajustés pour LAI $<6$ à une droite d'équation:

$$
\frac{1 \varphi}{t}=-0,15 \mathrm{LAI}+1
$$

Sur la même figure, nous avons mentionné des résultats empruntés d'une part à ANDERSON (1969) pour le blé (Triticum aestivum L.) et, d'autre part, à KUMURA (1969) pour le soja (Glycine max (L.) Merrill).

On observe une variabilité importante entre les résultats et une discussion sera faite ultérieurement à partir des résultats de la modélisation proposée.

- Divers paramètres biologiques étaient suivis sur la culture, notamment la surface foliaire, déterminée à l'aide d'un planimètre optique, et la matière sèche produite par organe déterminée par prélèvements échelonnés.

- Les propriétés optiques de feuilles, pour les bandes correspondant au rayonnement utile à la photosynthèse (PAR) et au proche infra-rouge ont été déterminées au Laboratoire de Chimie biologique et de Photophysiologie (INA Paris-Grignon). 
Sous l'angle interception du rayonnement, un couvert de luzerne peut être considéré comme un volume de feuilles vertes, de hauteur sensiblement constante, situé plus ou moins haut au-dessus du sol au cours de la saison de végétation. Ceci peut expliquer les faibles variations observées entre les repousses; en effet, la différence de structure porte ensuite sur les tiges situées sous ce volume de feuilles. Un essai de simulation en supposant des feuilles à inclinaison verticale fait apparaître une modification du bilan de 5 p. 100 maximum en période estivale.

\section{B. Simulation du rayonnement intercepté}

Les résultats précédents nous ont conduits à retenir une relation unique pour décrire la variation $\varepsilon \varphi_{i}$ en fonction de l'indice foliaire.

Cette relation est la suivante:

$$
\varepsilon \varphi_{\mathrm{i}}=0,97 \times\left(1-\mathrm{e}^{-0,88 \mathrm{LAI}}\right)
$$

La précision sur une valeur moyenne estimée est de $\pm 0,02$.

Cependant une telle relation n'est que statistique et ne permet pas d'étudier l'influence de certains paramètres sur le rayonnement intercepté, aussi, parallèlement à cette approche globale, nous avons adapté un modèle de pénétration du rayonnement dans la végétation.

Nous avons retenu le modèle décrit par BONHOMME \& VARLET-GRANCHER (1977) ; nous n'expliciterons ici que les points ayant fait l'objet d'une étude spécifique à la luzerne. Le modèle utilisé est un modèle instantané ; aussi, pour obtenir des données journalières comparables aux données expérimentales, il fallait utiliser avec ce modèle, soit :

- des données de rayonnement incident diffus et direct instantanées et intégrer les résultats sur la journée,

- un modèle de simulation des rayonnements direct et diffus au cours de la journée.

Nous avons choisi la seconde solution et retenu une démarche similaire à celle de PERrIN de BRICHAMBAuT (1978) et DuRAND \& LEgRos (1981). Les données de départ sont le rayonnement global journalier et la duréc d'insolation Ss. Nous avons supposé en outre une insolation aléatoire au cours de la journée.

Nous avons, à la suite de nombreux auteurs et notamment de VARLET-GRANCHER et al. (1981) pour le site envisagé, considéré la valeur du rapport $P A R_{i} / G_{i}$ comme constante et égale à 2 pour le système d'unités défini dans le tableau 1 .

L'utilisation du modèle de pénétration du rayonnement nécessite de plus quelques paramètres biologiques spécifiques :

\section{Les propriétés optiques des feuilles}

La figure 5 représente la variation de la somme des coefficients de réflexion $\rho$ et de transmission $\tau$ en fonction de la longueur d'onde. Ces courbes, appliquées à 2 spectres naturels extrêmes (ciel clair, ciel couvert), conduisent pour $\rho+\tau$ à des coefficients pondérés de 0,18 en condition de ciel clair et de 0,15 en condition de ciel couvert, dans la gamme $400-700 \mathrm{~nm}$.

Le modèle utilisé suppose l'égalité des coefficients $\rho$ et $\tau$, bien que les mesures spectrophotométriques obtenues par divers auteurs montrent que la réflectance est toujours supérieure à la transmittance; aussi avons-nous de plus

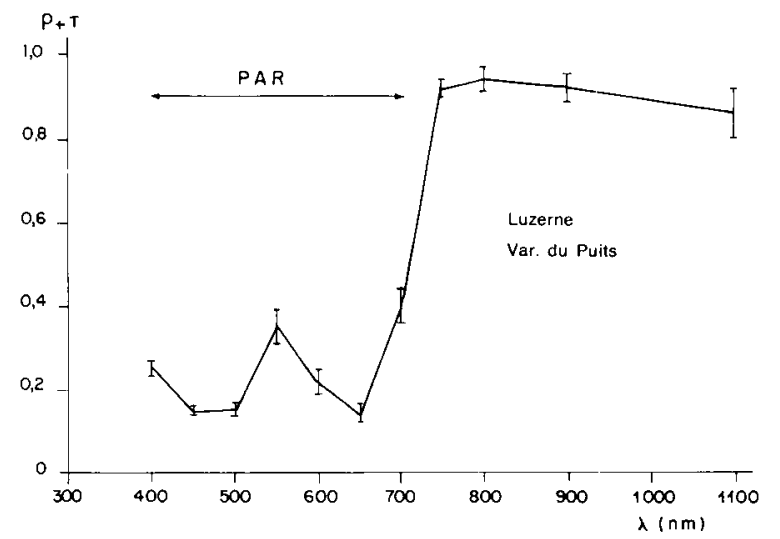

Figure 5

Propriétés optiques des feuilles de luzerne.

Optical properties of alfalfa leaves.

mesuré directement en conditions naturelles la transmittance ; nous avons obtenu :

$$
\tau=0,075 \pm 0,004
$$

La réflectance est donc supérieure de 15 p. 100 à la transmittance ce qui reste satisfaisant dans le cadre du modèle utilisé.

\section{L'inclinaison moyenne des feuilles}

Nous avons retenu, à la suite de SCOTT \& WELLS (1969), une inclinaison moyenne per rapport à l'horizontale de $35^{\circ}$.

\section{L'azimut des feuilles}

Bien que SCOTT \& WELLS (1969) signalent un effet azimut au cours de la journée, nous avons supposé une répartition isoazimut; en effet, d'une journée à l'autre, le comportement des feuilles doit être similaire et cet effet, qui peut être important en valeur instantanée, doit s'estomper sur des valeurs journalières.

\section{Superposition des feuilles}

La discussion relative à la hauteur photosynthétiquement active de la culture montre qu'elle existe mais qu'elle reste sensiblement la même pour les différentes coupes.

Nous n'avons pas cherché à tester l'ensemble des paramètres du modèle mais seulement les paramètres pouvant présenter une dynamique dans le cadre de cette application, aussi avons-nous retenu :

- l'indice foliaire LAI,

- le type de temps caractérisé par le rapport Diffus/Global avec 2 conditions extrêmes $(D / G=0,18$ ciel clair ; $\mathrm{D} / \mathrm{G}=1$ ciel couvert),

- la période de l'année, afin de tester l'influence de la hauteur du soleil par rapport à l'inclinaison moyenne des feuilles (nous avons choisi 3 dates : $1^{\text {er }}$ mai, $1^{\text {er }}$ juillet, $1^{\text {er }}$ novembre).

Les résultats présentés ont trait aux paramètres suivants ;

- la réflectance $r \varphi$ pour le rayonnement photosynthétiquement actif,

- l'efficience d'interception $\varepsilon \varphi_{\mathrm{i}}$,

- le rapport $t \varphi / t$.

a) Pour la réflectance $r \varphi$, les résultats obtenus sont similaires à ceux trouvés dans la littérature :

- pour des indices foliaires faibles $(\mathrm{LAI}<2)$, le rôle de la réflectance du sol $\mathrm{r} \varphi_{\mathrm{s}}$ est déterminant, 
- pour des indices foliaires supérieurs à 2 , la réflectance de la culture est constante, égale à 0,04 . L'adéquation entre nos données expérimentales et les résultats de SHEEHY et al. (1979) est bonne. Par ailleurs cette réflectance présente une faible variation vis-à-vis du type de temps et du moment dans l'année.

$b)$ L'efficience d'interception $\varepsilon \varphi_{\mathrm{i}}$ présente une faible variation vis-à-vis des paramètres retenus (fig. 6), il existe en outre une bonne concordance avec les points expérimentaux quel que soit l'indice foliaire. Néanmoins, ces résultats ne sont pas en parfait accord avec des travaux plus anciens, de HuNT et al. (1970) notamment; en effet ces auteurs mettent en évidence des variations interpousses. BROWN et al. (1972) interprètent ces différences comme étant dues à des variations selon les conditions climatiques de :

- l'azimut des feuilles,

- l'inclinaison des feuilles.

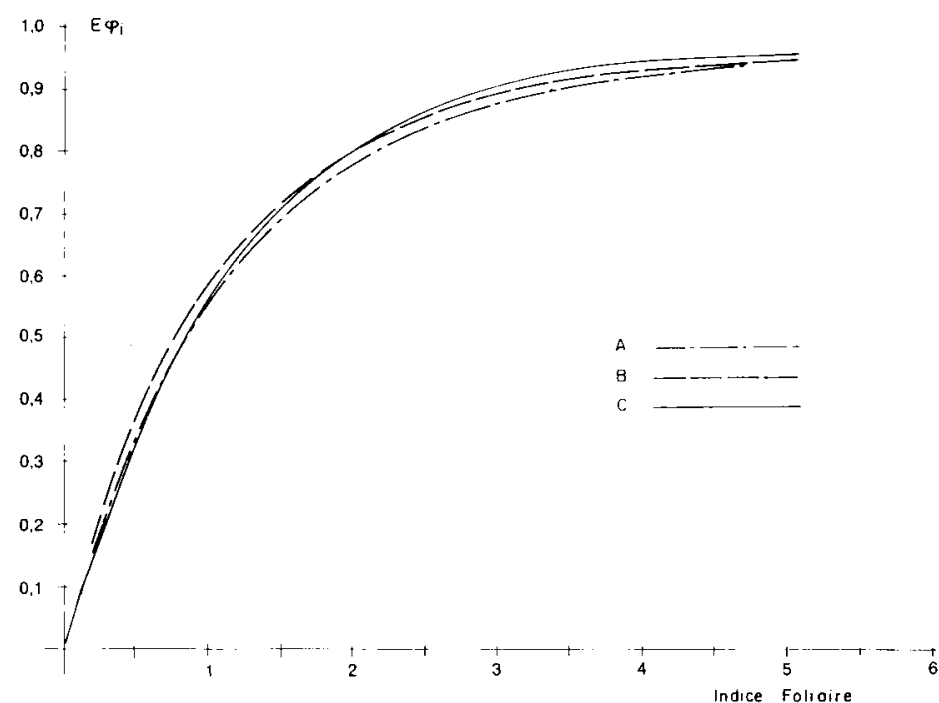

Figure 6

Evolution de l'efficience d'interception $\varepsilon \varphi_{i}$ simulée en fonction de l'indice foliaire.

La courbe $A$ regroupe les résultats de simulation pour les conditions: $1^{\text {er }}$ mai (ciel clair, ciel couvert), $1^{\text {er }}$ juillet (ciel clair, ciel couvert), $I^{\text {er }}$ novembre (ciel couvert).

La courbe $B$ correspond aux conditions de simulation $1^{\text {er }}$ novembre, ciel clair.

La courbe $C$ représente l'ajustement des points expérimentaux (relation 2).

Simulated solar trapping efficiency in relation to leaf area index : Curve A : Data from May 1st (clear and overcast sky). July 1st (clear and overcast sky), November Ist (overcast sky).

Curve B: Data from November 1st (clear sky).

Curve $C$ : Fitted from experimental data (equation 2).
Les seules données disponibles (SCOTT \& WELLS, 1969) ne permettent pas d'étayer ces hypothèses. Par ailleurs ces résultats sont obtenus à partir de mesures instantanées (à 12 h T.U.) avec des capteurs anthracène sensibles pour des longueurs d'ondes variant de 300 à $400 \mathrm{~nm}$ et supposant une pénétration du rayonnement utile à la photosynthèse similaire au rayonnement ultraviolet. La trop grande disparité des techniques ne nous permet pas de déterminer si la différence entre les résultats de HUNT et al. (1970) et les nôtres est due aux techniques utilisées par HuNT et al. (1970) ou à un comportement différent du couvert de luzerne entre les 2 sites.

c) Afin d'expliquer les divergences obtenues sur les rapports $t \varphi / t$ (fig. 1), nous avons, pour différentes inclinaisons de feuilles par rapport à l'horizontale $\left(10^{\circ}, 35^{\circ}, 50^{\circ}\right.$ et $70^{\circ}$ ), simulé la valeur de ce rapport pour une gamme d'indice foliaire variant de 0 à 5 . Les points obtenus par Kumura (1969) sur soja s'ajustent bien aux courbes $20-35^{\circ}$; cette inclinaison correspond au port peu dressé du soja. Les points obtenus sur luzerne s'ajustent autour de la courbe $50^{\circ}$; compte tenu des incertitudes, d'une part sur le modèle et d'autre part sur les mesures, il est impossible de donner une explication à cette faible divergence. Par contre, l'ajustement obtenu par ANDERSON (1969) conduit à une surestimation systématique du rapport $t \varphi / t$.

Nous avons cependant testé l'incidence de ces différentes façons d'estimer le rapport $t \varphi / t$ sur l'efficience d'interception $\varepsilon \varphi_{i}$, calculée pour un cycle de végétation (VARLETGrancher et al., 1982). Pour l'année 1979 et dans nos conditions expérimentales, le tableau 2 fait apparaître une sous-estimation systématique de l'ordre de $6 \mathrm{p} .100$ pour la relation retenue par VARLET-GRANCHER et al. (1982) et une surestimation de $4 \mathrm{p}$. 100 pour le modèle par rapport à la relation [1] proposée. Il est néanmoins difficile de généraliser ; en effet pour des périodes de végétation aussi courtes (de l'ordre de $40 \mathrm{j}$ ), la répartition du rayonnement incident au cours de cette période peut provoquer des distorsions de l'ordre de grandeur de celles induites par le mode de calcul.

\section{CONCLUSION}

Dans le cadre de cette étude, nous avons montré que le type de repousse (printemps, été, automne) n'influence pas, de par la structure du couvert, la part de rayonnement intercepté : une relation unique - relation (2) - entre l'efficience d'interception et l'indice foliaire a donc été proposée comme modélisation globale du rayonnement intercepté par une luzerne.

TABLEAU 2

Effet du mode d'estimation du rapport $\mathrm{t}_{\varphi} / \mathrm{t}$ sur l'cfficience d'interception $\varepsilon \varphi_{\mathrm{i}}$ calculée pour un cycle de végétation. Effect of different ways of estimation of the ratio $t \varphi / t$ on solar trapping efficiency $\varepsilon \varphi_{i}$ over a growth period.

\begin{tabular}{|c|c|c|c|c|}
\hline Année 1979 & $\begin{array}{c}\text { Pousse de printemps } \\
\text { J. } 79-147\end{array}$ & $\begin{array}{l}1^{\mathrm{re}} \text { repousse } \\
\text { J. } 148-193\end{array}$ & $\begin{array}{l}2^{\mathrm{e}} \text { repousse } \\
\text { J. } 194-235\end{array}$ & $\begin{array}{l}3^{\mathrm{c}} \text { repousse } \\
\text { J. } 236-282\end{array}$ \\
\hline $\begin{array}{l}\text { Avec la relation proposée par } \\
\text { M. ANDERSON }\end{array}$ & 0,62 & 0,68 & 0,50 & 0,53 \\
\hline Avec la relation (1) & 0,66 & 0,72 & 0,54 & 0,57 \\
\hline $\begin{array}{l}\text { Avec les résultats du modèle (inclinai- } \\
\text { son } 35^{\circ} \text { ) }\end{array}$ & 0,68 & 0,74 & 0,56 & 0,60 \\
\hline
\end{tabular}


L'explication de la différence de comportement entre les repousses d'une luzerne dans nos conditions est à rechercher ailleurs :

- conditions de rayonnement incident différentes,

- réponse photosynthétique différente,

- vitesse d'établissement de la surface foliaire et répartition des assimilats différentes.

L'étude annexe menéc sur le rapport $t \varphi / t$ montre que si l'on étudie l'efficience d’interception sur des périodes de temps longues, le choix de la relation entre $t \varphi / t$ et l'indice foliaire n'est pas déterminant; par contre, sur des laps de temps courts, ce choix peut devenir prépondérant.

La bonne concordance entre les résultats fournis par le modèle analytique et les données expérimentales fait de ce modèle un bon outil d'analyse que l'on peut intégrer dans des analyses de production.

Les deux voies de simulation proposées répondent à des aspirations différentes :

- modélisation globale avec, dès à présent, deux applications : étude de l'efficience énergétique d'une culture sur des cycles de longue durée (VARLET-GRANCHER et al., 1982), description du bilan de carbone en phase diurne grâce au rayonnement intercepté et application à la simulation de la production de matière sèche (HODGES \& KANEMASU, 1977 ; GoSSE et al., 1981);

- modélisation plus analytique, d'après BONHOMME \& VARLET-GRANCHER (1977) : cette démarche permet, outre la description des composantes radiatives aux bornes du système culture, une description du profil de rayonnement dans. la végétation. Ce profil de rayonnement est alors utilisé dans le cadre d'un modèle de photosynthèse de la culture (CHARTIER, 1969).

Cette démarche est utile pour expliquer les variations observées dans la répartition du carbone entre les feuilles et les tiges au cours d'une saison de végétation.

Les deux approches sont réalisées simultanément et confrontées aux données expérimentales (travaux en cours de rédaction).

Reçu le 15 décembre 1981. Accepté le 8 mars 1982.

\section{RÉFÉRENCES BIBLIOGRAPHIQUES}

Allen W. A., Richardson A. J., 1968. Interaction of light with a plant canopy. J. Opt. Soc. Amer., 58, 1023-1028.

Anderson M. C., 1969. A comparison of two theories of scattering of radiation in crops. Agric. Meteorol., 6, 399-405.

Bonhomme R., Varlet-Grancher C., 1977. Application aux couverts végétaux des lois de rayonnement en milicu diffusant. I. Etablissement des lois et vérifications expérimentales. Ann. agron., 28 (6), 567-582.

Brown R. H., Pearce R. B., Wolf D. D., Blaser R. E., 1972. Energy accumulation and utilization. In: «Alfalfa science and techno$\log y »$, Hanson éd. American Society of Agronomy, $\mathrm{n}^{\circ} 15$, p. 143164 .

Chartier P., 1969. Assimilation nette d'une culturc couvrante. I. Détermination de l'assimilation nette de la culture à partir d'une analyse théorique. Ann. Physiol. vég., 11 (2), 123-159.

Demarly Y., 1957. Biologie et exploitation de la luzerne. Ann. Amélior. Plantes, 3, 247-272.

Durand R., Legros J. P., 1981. Cartographic automatique de l'énergie solaire en fonction du rclief. Agronomie, 1, 31-40.

Gosse G., Chartier M., Mauget C., Fiala V., Bethenod O., 1981. Net $\mathrm{CO}_{2}$ exchange and dry matter production of alfalfa. $O E C D$ Conf. Ettlingen, Octobre 1981.
Hodges T., Kanemasu E. T., 1977. Modeling daily dry matter production of winter wheat. Agron. J., 69, 974-978.

Hunt L. A., Moore C. E., Winch J. E., 1970. Light attenuation coefficient and productivity in "vernal " alfalfa. Can. J. Plant Sci., 50, 469-474.

Kumura A., 1969. Studies on dry matter production of soybean plant. VI. Changes in spectral composition of solar radiation penetrating through leaf canopy and photosynthetic rate of single leaf as affected by light quality. Proceed. Crop Sci. Soc. Japan, 38, 408-418.

Perrin de Brichambaut, 1978. Estimation de l'énergie solaire disponible au sol. La Météorologie, $\mathrm{VI}^{\mathrm{c}}$ série, 15, 5-45.

Scott D., Wells J. S., 1969. Leaf orientation in barley, lupin and lucerne stands. N.Z. J. Bot., 7, 372-388.

Sheehy J. E., Woodward F. J., Jones M. B., Windram A., 1979. Microclimatc photosynthesis and growth of lucerne. I. Microclimate and photosynthesis. Ann. of Bot., 44, 693-707.

Varlet-Grancher C., Bonhomme R., Chartier M., Artis P., 1982. Efficience de la conversion de l'énergie solaire par un couvert végétal. Oecol. Plant., 3 (17), 3-26.

Varlet-Grancher C., Chartier M., Gosse G., Bonhomme R., 1981. Rayonnement utile pour la photosynthèse des végétaux en conditions naturelles. Oecol. Plant., 2 (16), 189-202. 